\title{
INTERCONSULTA DE ENFERMAGEM PSIQUIÁTRICA: ANÁLISE DA PRODUÇÃO CIENTÍFICA
}

Lídia Neusa de Freitas Glanzmann', Vanessa Pellegrino Toledo²

RESUMO: Trata-se de revisão integrativa da literatura com o objetivo de analisar a publicação, em periódico científico, sobre interconsulta de enfermagem psiquiátrica, enfocando base de dados de indexação, periódico, ano de publicação, delineamento metodológico e temática abordada. A busca dos artigos se deu nas bases de dados PubMed, MEDLINE e LILACS, com descritor interconsulta de enfermagem psiquiátrica e publicação no período de 1999 a 2010. Selecionaram-se dez artigos, a análise permitiu a identificação de que $50 \%$ dsesses estão indexados no PubMed, 20\% se encontram no periódico Archives of a Psychiatric Nursing, 20\% no International Journal of Mental Health Nursing e 20\% em Perspectives in Psychiatric Care, sendo de 2008 a maioria das publicações; $40 \%$ foram estudos do tipo qualitativo/ etnográfico. As temáticas mais prevalentes foram aplicação da interconsulta de enfermagem psiquiátrica em pacientes e opinião dos enfermeiros. Os resultados mostram a necessidade de mais estudos sobre o tema no Brasil e no mundo.

PALAVRAS-CHAVE: Enfermagem psiquiátrica; Referência e consulta; Saúde mental.

\section{CONSULTATION-LIAISON PSYCHIATRIC NURSING: AN ANALYSIS OF SCIENTFIC OUTPUT}

\begin{abstract}
This integrative literature review aimed to analyze what was published in scientific periodicals on the subject of consultationliaison psychiatric nursing, focussing with a database of indexing, periodical, year of publication, methodological delineation and topic addressed. The article search was done with the PubMed, MEDLINE and LILACS databases, using the descriptor consultation-liaison psychiatric nursing and publication in the period 1999-2010. Ten articles were selected - the analysis permitted the identification that $50 \%$ of these were indexed in PubMed, 20\% were found in the periodical Archives of Psychiatric Nursing, 20\% in the International Journal of Mental Health Nursing and 20\% in Perspectives in Psychiatric Care. The majority of publications were from 2008; $40 \%$ were qualitative or ethnographic studies. The most prevalent topics were application of consultation-liaison psychiatric nursing in patients and the opinion of nurses. The results show the necessity for more studies on the topic in Brazil and elsewhere in the world.
\end{abstract}

KEYWORDS: Psychiatric nursing; Reference and consultancy; Mental health.

\section{INTERCONSULTA DE ENFERMERÍA PSIQUIÁTRICA: ANÁLISIS DE LA PRODUCCIÓN CIENTÍFICA}

RESUMEN: Esta es una revisión integrativa de la literatura cuyo objetivo fue analizar la publicación, en periódico científico, acerca de interconsulta de enfermería psiquiátrica, con base en datos de indexación, periódico, año de publicación, delineación metodológica y temática abordada. La búsqueda de los artículos ocurrió en las bases de datos PubMed, MEDLINEy LILACS, con descriptor interconsulta de enfermería psiquiátrica y publicación en el periodo de 1999 a 2010. Fueron elegidos diez artículos y el análisis permitió la identificación de que 50\% de eses están indexados en el PubMed, 20\% están en el periódico Archives of a Psychiatric Nursing, 20\% en el International Journal of Mental Health Nursing y 20\% en Perspectives in Psychiatric Care, siendo de 2008 la mayoría de las publicaciones; $40 \%$ fueron estudios del tipo cualitativo/etnográfico. Las temáticas más prevalentes fueron aplicación de la interconsulta de enfermería psiquiátrica en pacientes y opinión de los enfermeros. Los resultados muestran la necesidad de más estudios sobre el tema en Brasil y en el mundo. PALABRAS-CLAVE: Enfermería psiquiátrica; Referencia y consulta; Salud mental.

\footnotetext{
${ }^{1}$ Acadêmica de Enfermagem pela Faculdade de Ciências Médicas da Universidade Estadual de Campinas-UNICAMP.

${ }^{2}$ Enfermeira. Doutora em Enfermagem. Professora do Departamento de Enfermagem da Faculdade de Ciências Médicas da UNICAMP.
}

Autor correspondente:

Vanessa Pellegrino Toledo

Universidade Estadual de Campinas

Avenida Dr. Lauro Correia da Silva, 3805 - 13481-631 - Limeira-SP-Brasil

E-mail: vtoledo@fcm.unicamp.br
Recebido: 14/01/2010

Aprovado: 07/06/2011

Cogitare Enferm. 2012 Jan/Mar; 17(1):138-43 


\section{INTRODUÇÃO}

$\mathrm{Na}$ atualidade, como resultado das políticas de saúde mental, existem unidades de psiquiatria em hospitais gerais ${ }^{(1)}$. Contudo, o atendimento de enfermagem a pacientes com doenças físicas configura-se pela execução de procedimentos altamente técnicos, disponíveis para a preservação da vida; e a equipe multiprofissional envolvida na assistência tende a priorizar a saúde física, deixando, muitas vezes, de abordar os aspectos emocionais do paciente ${ }^{(2)}$.

Diante disto, a compreensão da problemática "suporte emocional" ao paciente com doenças físicas pode ser facilitada com a consultoria psiquiátrica, realizada por meio da interconsulta de enfermagem psiquiátrica, que integra processos fisiológicos, bioquímicos e seus distúrbios, considerando os três âmbitos: biológico, psicológico e social ${ }^{(3)}$.

Para assistir aos pacientes com necessidades de cuidados psiquiátricos durante a internação em outras especialidades, tem-se a interconsulta de enfermagem psiquiátrica ou a psiquiatria de consultoria e ligação, que indica o conjunto de atividades desempenhadas pela enfermeira psiquiátrica junto às diferentes especialidades do hospital geral ${ }^{(4)}$.

A psiquiatria de consultoria e ligação de enfermagem (PCLN: Psychiatry Consultation-Liaision Nursing), é denominada no Brasil interconsulta de enfermagem psiquiátrica e consta de uma área avançada da Enfermagem Psiquiátrica e data dos anos 60 do século XX, trinta anos após o surgimento da psiquiatria de consultoria e ligação na Medicina ${ }^{(5)}$. Essa prática é comum em países de primeiro mundo, principalmente nos Estados Unidos e na Austrália, fato que explica a existência de poucos estudos sobre a interconsulta de enfermagem psiquiátrica no Brasil.

O termo liaison (ou ligação) é usado para representar vários aspectos da prática da PCLN. Denomina uma inter-relação entre a base do conhecimento de enfermagem psiquiátrica e de saúde mental e o cuidado aos pacientes com um atual ou potencial problema na saúde mental ${ }^{(6)}$. Também se refere a interação e relacionamento entre o enfermeiro psiquiátrico e a equipe de serviços não-psiquiátricos, com o foco em assistir a essa equipe e em reconhecer, manejar e prevenir problemas psiquiátricos e psicossociais em pacientes de hospitais gerais ${ }^{(7)}$.

O foco primário da PCLN está no âmbito emocional, no desenvolvimento espiritual e nas respostas cognitivas/ comportamentais dos pacientes/familiares que entram no sistema de cuidado de saúde com atual ou potencial disfunção psíquica; inclui consultas de enfermagem, colaboração na assistência prestada e também educação do paciente e da equipe como práticas diretas ${ }^{(7)}$.

A necessidade de enfermeiros psiquiátricos interconsultores vem aumentando pelo incremento da especialização entre profissionais de saúde, especificamente da Enfermagem. Isto tem implicações diretas sobre a especificidade de cuidados nas distintas áreas de assistência, na crescente profissionalização da Enfermagem e com a necessidade dos enfermeiros resolverem problemas referentes à concepção de seu papel no contexto do seu marco profissional ${ }^{(5)}$. Porém, enfermeiros que trabalham em setores não psiquiátricos de hospitais gerais relatam falta de conhecimento, habilidade e confiança na assistência e manejo do paciente com problemas psíquicos e emocionais ${ }^{(4)}$.

Este estudo justifica-se pela escassa produção do conhecimento acerca da prática da interconsulta de enfermagem psiquiátrica em hospitais gerais do Brasil ${ }^{(2)}$, destacando os recentes avanços nas políticas de saúde mental, além da necessidade de aprendizado dos enfermeiros que assistem pacientes internados em unidades não psiquiátricas e seus familiares para compreensão mais ampliada sobre o tema, auxiliando pacientes que podem estar em risco de apresentar distúrbios mentais graves.

Considerando os aspectos mencionados no texto, o objetivo deste estudo é analisar a publicação em periódico cientifico sobre interconsulta de enfermagem psiquiátrica.

\section{MÉTODO}

Trata-se de uma revisão bibliográfica integrativa sobre interconsulta de enfermagem psiquiátrica. A revisão integrativa permite ao pesquisador que sintetize elementos empíricos da literatura teórica sobre um tema específico, envolvendo algumas etapas que precisam ser seguidas ${ }^{(8)}$.

As etapas desenvolvidas para o alcance do objetivo foram: identificação do problema (elaboração da pergunta norteadora, estabelecimento de descritores e de critérios para inclusão/exclusão de artigos), seleção dos artigos, definição das informações a serem extraídas dos trabalhos revisados (objetivos, metodologia e principais conclusões), bem como análise, discussão e interpretação dos resultados e, por fim, síntese do conhecimento $^{(8)}$.

A pergunta norteadora do estudo foi "Qual a produção científica existente sobre a interconsulta de enfermagem psiquiátrica?" Os critérios de inclusão das 
publicações foram: artigos que tratassem da temática em estudo como foco central, publicações em inglês e português publicados no período de 1999 a 2010 e que estivessem com os resumos disponíveis e indexados nas bases PUBMED arquivo digital produzido pela National Library of Medicine - USA, na área das Biociências, LILACS (Latin-American and Caribbean Health Sciences) e MEDLINE (Medical Literature and Retrieval System on Line).

Utilizou-se como descritor "interconsulta de enfermagem psiquiátrica" (nursing consultation liaison psychiatry) no DeCS e MESH.

Para a seleção das publicações, os títulos e resumos foram lidos exaustivamente, confirmando se contemplavam a pergunta norteadora deste estudo e se corroboravam com os critérios de inclusão estabelecidos.

Foram encontrados 14 artigos e a análise dos dados foi realizada a partir da leitura dos mesmos, sendo que 10 foram selecionados para este estudo, baseando-se nos critérios de inclusão. Dessa forma, cada artigo foi classificado de acordo com a base de dados em que estava indexado, o periódico em que estava publicado, o ano de publicação, o delineamento metodológico empregado pelos autores e a temática abordada. Para subsidiar a análise do delineamento metodológico utilizou-se o referencial descrito através das categorias de estudos quantitativos (experimental, quase-experimental e não-experimental) equalitativos (etnografia, fenomenologia e teoria fundamentada) ${ }^{(9)}$. Utilizou-se a planilha do programa computacional Excel para a inserção dos dados.

\section{RESULTADOS}

$\mathrm{Na}$ análise das publicações percebe-se que uma pequena porcentagem dos estudos foi desenvolvida no Brasil. Evidenciou-se as bases de dados: cinco PUBMED (50\%), três MEDLINE (30\%), um PUBMED e MEDLINE (10\%), e um $(10 \%)$ LILACS.

Os periódicos que publicaram os artigos e frequencia estão apresentados na tabela 1 .
A tabela 2 mostra que a maioria dos artigos foram publicados no ano de 2008. O delineamento metodológico dos artigos está apresentado na tabela 3.

Tabela 2 - Distribuição de artigos publicados sobre interconsulta de enfermagem psiquiátrica e ano da publicação, 1999-2010. Campinas, 2011

\begin{tabular}{lcc}
\hline \multicolumn{1}{c}{ ANO } & N & \% \\
\hline 1999 & - & - \\
2000 & - & - \\
2001 & 01 & 10 \\
2002 & 01 & 10 \\
2003 & - & - \\
2004 & 01 & 10 \\
2005 & - & - \\
2006 & 02 & 20 \\
2007 & - & 0 \\
2008 & 03 & 30 \\
2009 & 01 & 10 \\
2010 & 01 & 10 \\
Total & 10 & 100 \\
\hline
\end{tabular}

Tabela 3 - Distribuição de artigos publicados sobre interconsulta de enfermagem psiquiátrica de acordo com o delineamento metodológico, 1999-2010. Campinas, 2011

\begin{tabular}{lcc}
\hline DELINEAMENTOMETODOLÓGICO & N & \% \\
\hline Qualitativo - etnográfico & 04 & 40 \\
Qualitativo - fenomenológico & 03 & 30 \\
Qualitativo - teoria fundamentada & 03 & 30 \\
Total & 10 & 100 \\
\hline
\end{tabular}

As temáticas abordadas nos artigos publicados sobre interconsulta de enfermagem psiquiátrica estão apresentadas na tabela 4. É importante ressaltar que um dos estudos apresentou duas das temáticas, sendo contado duas vezes, pois além do trabalho aplicar a PCLN em pacientes, também ouviu a opinião dos enfermeiros sobre o tema.

Tabela 1 - Distribuição de periódicos científicos e artigos publicados sobre interconsulta de enfermagem psiquiátrica, 1999-2010. Campinas, 2011

\begin{tabular}{lll}
\hline \multicolumn{1}{c}{ PERIÓDICO } & N & \% \\
\hline Contemporary Mental Health Nursing & 01 & 10 \\
Archives of a Psychiatric Nursing & 02 & 20 \\
Australian Psychiatry & 01 & 10 \\
Internacional Journal of Mental Health Nursing & 02 & 20 \\
Perspectives in Psychiatric Care & 02 & 20 \\
Revista Latino -Americana de Enfermagem & 01 & 10 \\
Medical Suisse & 01 & 10 \\
Total & 10 & 100 \\
\hline
\end{tabular}

Cogitare Enferm. 2012 Jan/Mar; 17(1):138-43 
Tabela 4 - Temáticas abordadas em artigos publicados sobre interconsulta de enfermagem psiquiátrica, 1999-2010. Campinas, 2011

\begin{tabular}{lcc}
\hline \multicolumn{1}{c}{ TEMÁTICA } & N & $\mathbf{\%}$ \\
\hline Aplicação da PCLN* em pacientes & 03 & 30 \\
Opinião dos enfermeiros sobre a PCLN* & 03 & 30 \\
Opinião dos pacientes e/ou familiares sobre a PCLN* na residência & 01 & 10 \\
Avalia o conhecimento das enfermeiras sobre a PCLN* & 02 & 20 \\
Delimita a função da enfermeira na PCLN* & 01 & 10 \\
Aplicação da PCLN* a soldados americanos em guerra & 01 & 10 \\
Total & 11 & 110 \\
\hline *PCLN: Psychiatry Consultation-Liaision Nursing
\end{tabular}

\section{DISCUSSÃO}

A interconsulta de enfermagem psiquiátrica é considerada uma área especial de conhecimento que se ocupa da assistência, ensino e pesquisa na interface da Enfermagem Psiquiátrica. No Brasil, o número de serviços de interconsulta de enfermagem psiquiátrica é desconhecido e sua quase totalidade encontra-se em hospitais-escola. Apesar de já estar definido o papel do enfermeiro psiquiátrico interconsultor nos Estados Unidos da América, identificando-o como uma subespecialidade da Enfermagem Psiquiátrica e de Saúde Mental, e na Inglaterra haver maior atuação deste profissional na chamada interconsulta de ligação, no Brasil estudos demonstram que esta prática não tem, até o momento, sido proposta ou identificada ${ }^{(2)}$. Contudo, mesmo nos países em que essa prática é utilizada, pode-se afirmar que poucos estudos sobre o assunto estão publicados, considerando que a literatura disponível mostra a escassez das pesquisas sobre o tema ${ }^{(10)}$. Por outro lado, é relevante ressaltar o aumento do número de pesquisas sobre PCLN com o passar dos anos, a presente revisão revela aumento do número de estudos nos anos de 2006 e 2008.

A análise do delineamento metodológico das publicações estudada corrobora os achados da literatura, que destacam a abordagem metodológica qualitativa como amplamente utilizada em diversas áreas do conhecimento e também na Enfermagem ${ }^{(11)}$. Autores identificaram que $50 \%$ das pesquisas publicadas no período de 1977 a 2001 apresentaram delineamento qualitativo, sendo a fenomenologia o mais utilizado ${ }^{(12)}$. Ainda em relação ao delineamento metodológico faz-se necessário compreender a associação de sua escolha com o objeto a ser estudado e não à preferência do pesquisador $^{(13)}$. Ou seja, dependendo do objeto estudado, são identificadas variações sobre o delineamento metodológico empregado ${ }^{(13)}$.
No cenário das pesquisas qualitativas, ao optar por um método e suas técnicas de coleta, incluindo o tratamento dos dados, é importante que o pesquisador obtenha uma visão global dos resultados, dando ênfase ao corpus que compreende as observações e as entrevistas ${ }^{(13)}$.

As temáticas abordadas nas publicações que fizeram parte deste estudo retratam e reafirmam a falta de pesquisas sobre o tema, pois a maioria dos artigos aplica a interconsulta de enfermagem psiquiátrica em pacientes internados em unidades não psiquiátricas de hospitais gerais, avalia o conhecimento das enfermeiras sobre o assunto ou observa as opiniões a respeito da PCLN, ou seja, demonstram uma falta de conhecimento dos enfermeiros, e até mesmo dos pacientes, sobre a existência deste tipo de interconsulta. A maioria dos artigos experimenta a PCLN e enaltece a necessidade de mais estudos para fundamentarem esta prática. Ao mesmo tempo, as pesquisas sobre PCLN envolvem estágios conceituais, por meio do reflexo do desenvolvimento profissional desta subespecialidade, e não demonstram como esta temática pode ser implementada na prática ${ }^{(10)}$.

Um estudo realizado nos Estados Unidos demonstrou que $40 \%$ das internações em hospitais gerais acontece com pacientes acima de 65 anos, 25\% a $45 \%$ destes desenvolvem depressão (maior e menor) e de $14 \%$ a $56 \%$ desenvolvem delirium ${ }^{(14)}$. Há grande necessidade de interconsulta de enfermagem psiquiátrica em hospitais gerais pelo número de pacientes que desenvolvem doenças, como transtornos de humor, distúrbios psicóticos, cognitivos, de personalidade e até mesmo por substâncias utilizadas em sua recuperação ${ }^{(7)}$. E, na maioria das vezes, os profissionais de saúde que trabalham nestas unidades não possuem o conhecimento específico em psiquiatria, dificultando a recuperação dos pacientes e até mesmo a percepção de seus sintomas. A fragmentação do cuidado e a desresponsabilização pelo paciente é constituída pela condição estática e departamentalizada do processo 
condutor da terapêutica e constituem um empecilho ao desenvolvimento de condutas emancipatórias ${ }^{(15)}$.

Particularmente, enfermeiros que trabalham em unidades não psiquiátricas de hospitais gerais referem um deficit no conhecimento, habilidade e confiança na assistência e no manejo de pacientes com problemas psíquicos e emocionais. Sentimentos como medo, inaptidão e incompreensão foram identificados na rotina desses profissionais ${ }^{(16)}$.

\section{CONSIDERAÇÕES FINAIS}

A interconsulta de enfermagem psiquiátrica caracteriza-se como importante ferramenta para assistir pacientes internados em hospitais gerais e, até mesmo, aos que estão sob cuidados da enfermagem domiciliar, frente a mudanças no estilo de vida pessoal, nos relacionamentos familiares e sociais, bem como alterações na autoimagem e no autoconceito do indivíduo. Muitos sintomas ou transtornos psíquicos podem aparecer como comorbidades em decorrência de outras doenças, as quais podem mudar, ou mesmo limitar, as atividades cotidianas de pessoas comuns.

Neste estudo confirmou-se que a temática mais abordada é a aplicação da PCLN, sendo mais empregado nas pesquisas, porém ainda pouco aplicada nos serviços de saúde. A falta de conhecimento sobre a PCLN, ou mesmo sobre os distúrbios psiquiátricos e o manejo desses pacientes por enfermeiros de outras áreas em hospitais gerais, também foi demonstrado neste estudo, pois na maioria das publicações em que esse tema foi abordado observou-se o despreparo ou mesmo o medo dos enfermeiros ao lidar com pacientes que apresentam sintomas psíquicos ou emocionais.

Para que prática da interconsulta de enfermagem psiquiátrica possa ser realizada de maneira efetiva é necessária a valorização da atuação do enfermeiro psiquiátrico em hospital geral, assim como há a necessidade de mais estudos sobre este tema no Brasil e no mundo, principalmente no que se refere àqueles que apliquem este conhecimento.

\section{REFERÊNCIAS}

1. Botega NJ, Dalgalarrondo P. Saúde mental no hospital geral: espaço para o psíquico. São Paulo: Hucitec; 1993.

2. Scherer ZAP, Scherer EA, Labate RC. Interconsulta de enfermagem psiquiátrica: qual a compreensão do enfermeiro sobre esta atividade? Rev. latino-am. enfermagem. 2002;10(1):7-14.

3. Meleiro AMAS. Abordagem do psicótico no hospital geral: interconsulta psiquiátrica. In: Miguel Filho EC, Fráguas Junior R, Meleiro AMAS, Assumpção Junior FB, editores. Psiquiatria e psicologia no hospital geral: a clínica das psicoses. São Paulo: Mattavelli; 1992. p. 38-46.

4. Shavitt RG, Busato Filho G, Miguel Filho EC. Interconsulta psiquiátrica: conceito e evolução. Rev. paul Med. 1989;107(2):108-12.

5. Moschler LB, Fincannon J. Subspecialization within psychiatric consultation-liaison nursing. Arch. psychiatr. nurs. 1992;6(4):234-8.

6. American Nurses Association. Council on Psychiatric and Mental Health Nursing. Standards of psychiatric consultation-liaison nursing practice. Kansas City: American Nurses Association; 1990.

7. Scharrock J, Grigg M, Happel B, Keeble Devlin B, Jennings $S$. The mental health nurse: a valuable addition to the consultation-liaison team. Int. j ment. health nurs. 2006;15(1):35-43.

8. Whittemore R, Knafl K. The integrative review: updated methodology. J. adv. nurs. 2005;52(5):546-53.

9. Polit DF, Beck CT, Hungler BP. Fundamentos de pesquisa em enfermagem: métodos avaliação e utilização. $5^{\mathrm{a}}$ ed. Porto Alegre: Artmed; 2004.

10. Yakimo R, Kurlowicz LH, Murray RB. Evaluation of outcomes in psychiatric consultation-liaison nursing practice. Arch. psychiatr. nurs. 2004;18(6):215-27.

11. Demo P. Pesquisa qualitativa: busca de equilíbrio entre forma e conteúdo. Rev. latino-am. Enfermagem. 1998;6(2):89-104.

12. Oliveira EB, Lisboa MTL. Análise da produção científica da vertente saúde do trabalhador de enfermagem: subjetividade e trabalho. Rev. enferm. UERJ. 2004;12(1):24-9.

13. Campos CJG, Turato ER. Análise de conteúdo em pesquisas que utilizam metodologia clínico-qualitativa: aplicação e perspectivas. Rev. latino-am. Enfermagem. 2009;17(2):259-64.

14. Kurlowicz LH. Benefits os psychiatric consultationliaison nurse interventions for older hospitalized patients and their nurses. Arch. psychiatr. nurs. 2001;15(2):53-61. 
15. Pinto AGA, Jorge MSB. Prática clínica de saúde mental no cotidiano de um centro de atenção psicossocial. Cogitare enferm. 2009;14(2):217-26.

16. Bailey SR. An exploration of critical care nurses' and doctors' attitudes to parasuicide patients. Aust. j. adv. nurs. 1998;11(1):11-7. 